\title{
Hybrid Simulation Study on Cooperative Deformation of Nonlinear Steel-Cable Structure
}

\author{
Zhang Yong ${ }^{1,}$, Xu Zhihong ${ }^{2}$, Chen Jiahao ${ }^{1}$ \\ ${ }^{1}$ Department of Civil Engineering, Nanjing University of Science and Technology, Nanjing, China \\ ${ }^{2}$ School of Science, Nanjing University of Science and Technology, Nanjing, China
}

\section{Email address:}

zhyong11@163.com (Zhang Yong)

${ }^{*}$ Corresponding author

\section{To cite this article:}

Zhang Yong, Xu Zhihong, Chen Jiahao. Hybrid Simulation Study on Cooperative Deformation of Nonlinear Steel-Cable Structure. Science Discovery. Vol. 8, No. 5, 2020, pp. 101-106. doi: 10.11648/j.sd.20200805.15

Received: September 8, 2020; Accepted: October 10, 2020; Published: October 17, 2020

\begin{abstract}
In this paper, a hybrid simulation test method is used to study the cooperative deformation of nonlinear steel-cable structures. The general structural deformation analysis methods include the overall structure model test method and the numerical simulation method. The overall structure model test method is costly and the process is complicated; the numerical simulation method requires high requirements and requires prior knowledge of the constitutive relationship of the structure or component. The hybrid simulation test method combines the advantages of the two, that is, the entire structure is divided into two, one part is used as a test substructure for model testing, and the other is used as a numerical substructure for numerical simulation. In this study, the nonlinear cable is used as the experimental substructure for model test, and the rest of the cable is used as the numerical substructure for numerical simulation. Through the synchronous interactive feedback of the data between the two, the combined deformation of the nonlinear frame-type steel-cable structure is gradually obtained. Finally, the results of the hybrid simulation test are compared with the results of the numerical simulation test to verify the effectiveness of the hybrid simulation test method. The research in this paper can provide a new method for the combined deformation calculation of nonlinear steel-cable structures.
\end{abstract}

Keywords: Hybrid Simulation Test, Nonlinear Cable, Combined Deformation, Numerical Simulation Test, Nonlinear Steel-Cable Structure

\section{非线性钢-索结构协同变形的混合仿真研究}

张勇 ${ }^{1^{*}}$, 徐志洪 ${ }^{2}$, 陈嘉浩 ${ }^{1}$

1 南京理工大学土木工程系, 南京, 中国

2 南京理工大学理学院, 南京, 中国

邮箱

zhyong11@163.com（张勇）

摘要: 该文采用混合仿真试验方法研究了非线性钢-索结构的协同变形。通常结构的变形分析方法有整体结构模型试验 法和数值仿真法。整体结构模型试验法成本高, 过程复杂; 数值仿真法要求高, 需要事先知道结构或构件的本构关系。 混合仿真试验方法融合了二者的优点, 即将整个结构一分为二, 一部分作为试验子结构进行模型试验, 一部分作为数 值子结构进行数值仿真。在该研究中将非线性拉索作为试验子结构进行模型试验, 将拉索之外的剩余部分作为数值子 结构进行数值模拟。通过二者之间的数据同步交互反馈，逐步得到非线性框架式钢-索结构的组合变形。最后将混合仿 
真试验结果与数值仿真试验结果相对比, 验证了混合仿真试验方法的有效性, 该文研究可为非线性钢-索结构的组合变 形计算提供新方法。

关键词：混合仿真试验，非线性拉索，组合变形，数值仿真试验，非线性钢-索结构

\section{1. 引言}

索最早应用于古代的用藤、竹和绳等建造的悬索桥, 随着发展，索的材料用到了铁链、钢材等[1]。索的结构也 从桥梁应用到建筑中，例如索膜结构、索网幕墙和张弦梁 组合结构等[2]。索具有一定的非线性, 承受拉力, 在建筑 桥梁大跨度轻量化等方面扮演着越来越重要的角色。

钢-索结构是现代索结构应用的基本形式。通常钢-索 结构变形研究的方法包括整体结构模型试验和数值仿真 [3]。整体模型试验成本高, 但能得到每一时刻的真实变形; 数值仿真成本低, 但需要先做大量的模型试验得到准确的 力与位移解析关系, 然后才能用有限元软件进行模拟。然 而, 由于拉索的非线性, 有限元软件的模拟难以准确高效。 基于此, 可以采用模型试验与数值仿真同步相结合的方法, 即混合仿真试验方法。通过受拉索的局部模型试验与钢柱 间的作用交互反馈，实现钢-索结构的组合变形分析。

本文应用混合仿真试验方法, 借助于南京理工大学土 木工程与力学实验室现有设备及利用 $\mathrm{C}++$ 语言自行编写 的数据通讯软件 (PCS8000-Matlab-Interface, PMI) [4-6], 对由拉索及其两端钢圆柱杆构成的且受跨中竖向荷载作 用的类似框架式钢-索结构的组合变形进行分析。钢-索结 构模型如图1所示。同时为验证混合仿真试验方法的准确 性, 采用Matlab软件编写数值仿真计算程序, 运用模型试 验得到的拉索的力与位移关系计算该钢-索结构的组合变 形, 作为对照。模型中的索长 $1500 \mathrm{~mm}$, 粗 $4 \mathrm{~mm}$; 钢圆柱 杆高 $1800 \mathrm{~mm}$, 内径 $51 \mathrm{~mm}$, 外径 $57 \mathrm{~mm}$ 。钢材弹性模量取 $\mathrm{E}=206000 \mathrm{~N} / \mathrm{mm}^{2}$ 。

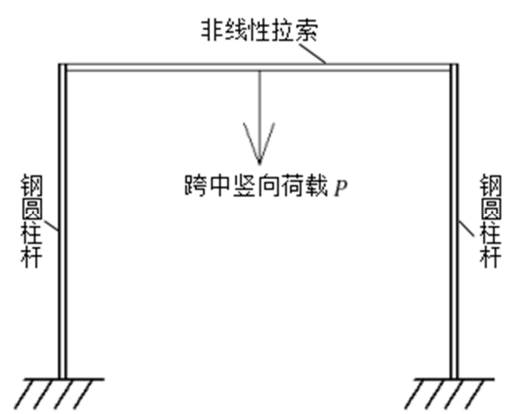

图1 钢-索结构模型。

\section{2. 混合仿真试验原理}

混合仿真试验将整个结构分为数值子结构和试验子 结构[7]。非线性拉索为试验子结构, 采用模型试验, 剩余 部分为数值子结构, 采用数值分析。二者通过分割处力、 位移相等考虑钢圆柱杆对拉索的约束作用 [8-10]。本文中 钢-索结构数值子结构与试验子结构的划分如图 2 所示。混 合仿真试验方法不依赖于整个结构, 因此大型且复杂的结 构系统也可以方便得到研究。

混合仿真试验过程可以划分为许多子步, 每个子步中 非线性拉索的刚度视为定值, 从而进行静力计算。首先通 过控制器控制试验加载设备向试验子结构施加初始位移 和速度, 由数据采集系统获取其反力。然后, 数据通讯软 件传递该反力到数值子结构中并由后者计算出下一子步 的位移和速度, 此为第一子步, 也即第一循环。该位移和 速度再次通过试验加载设备向试验子结构加载, 进入下一 子步, 也即下一循环。混合仿真试验原理如图3所示。

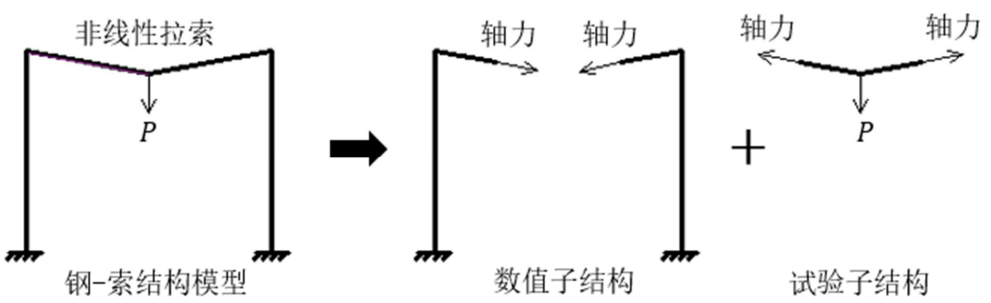

图2 钢-索结构数值子结构与试验子结构划分.

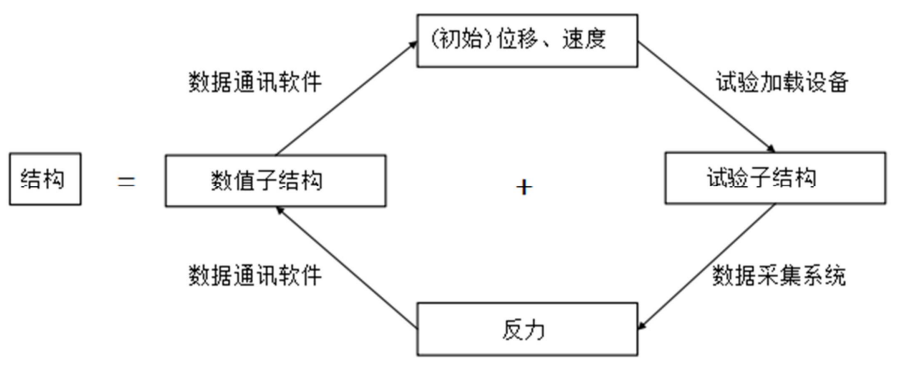

图3 混合仿真试验原理。 
每个子步计算的钢圆柱杆杆端水平位移即为该钢-索 结构的组合变形。文中进一步分析了索跨中挠度随荷载的 变化情况。本文不涉及动力学问题, 静力加载是通过每个 子步逐渐增加的, 在拉伸试验中不存在卸载行为。

\section{3. 混合仿真试验组成}

\section{1. 数值子结构}

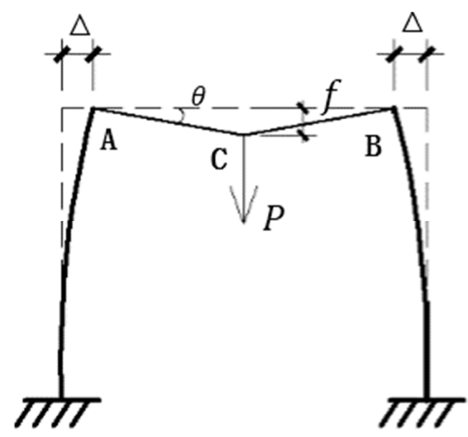

图4 几何非线性。

由于索只能轴向受拉, 所以该钢-索结构是具有一个 多余约束的超静定结构。在跨中坚向荷载作用下, 由于拉 索的的伸长变形以及圆柱杆端的位移, 杆端拉索的轴线方 向与水平方向夹角变化不容忽略, 如图4所示 ( $\Delta$ 为杆端
$\mathrm{A}$ 点的水平位移, $\theta$ 为杆端拉索轴线方向与水平方向的夹 角）。因此该结构不仅是材料非线性问题，而且是几何非 线性问题。本文考虑拉索的伸长变形以及夹角变化, 采用 虚功原理推导杆端 $\mathrm{A}$ 点水平位移 $(\Delta)$ 及拉索跨中挠度 $(f)$ 的计算公式 [11]。该结构为对称结构, 可简化为其半边结 构计算, 如图5所示。

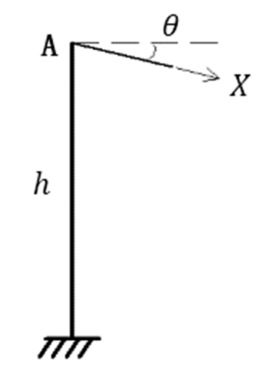

图5 结构对称简化模型。

首先不考虑拉索的伸长变形, 杆端 $\mathrm{A}$ 点的水平位移 $(\Delta)$ 等于拉索拉力作用下圆柱杆上的弯矩和剪力在 $\mathrm{A}$ 点 产生的水平位移之和。杆在拉索拉力作用下的弯矩图 $M_{p}$ 和剪力图 $F_{p}$ 以及单位力作用下的弯矩图 $\bar{M}$ 和剪力图 $\bar{F}$, 如 图6所示。



图6 拉索内力与单位力作用下的弯矩图和剪力图。

于是有:

$$
\begin{gathered}
\Delta M=\int \frac{M_{p} \bar{M}}{E I} d x=\frac{1}{E I} \int_{0}^{h} X \cos \theta \cdot x \cdot 1 \cdot x \cdot d x=\frac{X h^{3}}{3 E I} \cos \theta \\
\Delta F=k \cdot \int \frac{F_{p} \bar{F}}{G A} d x=\frac{2}{G A} \int_{0}^{h} X \cdot \cos \theta \cdot 1 \cdot d x=\frac{2 F h}{G A} \cos \theta \\
\Delta=\Delta M+\Delta F
\end{gathered}
$$

式中: $\Delta M$ 为弯矩作用下的水平位移; $\Delta F$ 为剪力作用 下的水平位移; $X$ 为拉索拉力 (未知, 由试验子结构反馈); $h$ 为圆柱杆高度; $E$ 为杨氏模量; $G$ 为剪切模量; $I$ 为截 面惯性矩; $A$ 为截面积; $\theta$ 为拉索轴线方向与水平方向的 夹角; $k$ 为切应变的截面形状系数 (当截面形状为薄壁圆 环形时, $k=2$ )。
然后考虑拉索的伸长变形, 因为 $\cos \theta$ 随着拉索的伸 长变形以及杆端水平位移 $\Delta$ (本文只考虑水平位移) 的变 化而变化, 如图4所示。有:

$$
\cos \theta=\frac{l-2 \Delta}{2\left(\frac{l}{2}+x\right)}=\frac{l-2 \Delta}{l+2 x}
$$

式中: $l$ 为拉索长度; $x$ 为拉索的变形量 (未知, 由 数值子结构计算得到);

将(4)式代入(1)(2)式, 再代入(3)式得杆端 $\mathrm{A}$ 点的水平 位移 $\Delta$ :

$$
\Delta=\frac{X l\left(h^{3}+2 h\right)}{(3 E I+G A) \cdot(l+2 x)+2 X\left(h^{3}+2 h\right)}
$$


根据图4的几何关系, 拉索跨中挠度 $f:$

$$
f=\left(\frac{l}{2}+x\right) \sin \theta
$$

(5)(6)式中包含未知的拉索拉力 $X$ 和拉索变形量 $x$ 。 在每个子步中, 由数值子结构计算得到 $x$, 由试验子结构 反馈得到 $X$ 。

最后由得到的 $X$ 根据跨中坚向荷载与索中拉力之间 的关系（即 $P-X$ 关系），如图7所示，可以得到跨中坚 向荷载 $P$ 。

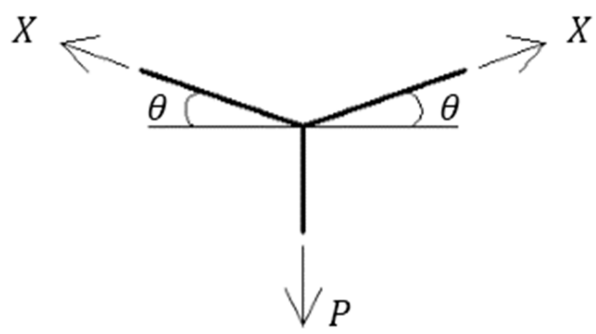

图7 跨中坚向荷载与拉索拉力的关系 ( $P-X$ 关系)。

即:

$$
\begin{aligned}
\sin \theta & =\sqrt{1-\cos \theta^{2}} \\
P & =2 X \cdot \sin \theta
\end{aligned}
$$

式中: $P$ 为拉索跨中坚向荷载; $X$ 为拉索拉力 (未 知, 由试验子结构反馈)。

至此即可建立跨中坚向荷载与圆柱杆端水平位移 $(\Delta)$ 即钢-索组合变形以及拉索跨中挠度 $(f)$ 的变化关系。

\section{2. 试验子结构}

本文试验加载设备采用瑞士Walter+Bai公司生产的 LFV-10型动态多功能测试设备, 如图8所示。其液压伺服
控制系统由 PCS 8000 控制器和DION7 控制软件组成。 PCS8000控制软件可以根据系统指令加载并反馈试验数 据, DION7 控制软件可以编辑用户自定义流程图, 实现特 定试验需求。实现计算机端及数值子结构与试验子结构数 据交换的软件是数据通讯软件PMI, 如图9所示。PMI由 $\mathrm{C}++$ 语言编写。数值子结构的Matlab计算程序用Matlab编 译器转化为 $\mathrm{C}++$ 语言代码内嵌在PMI中供其调用。

试验过程中, PMI将通过Matlab计算文件计算出的数 值子结构的位移和速度数据传递给PCS 8000 控制器, PCS8000控制器对试验子结构即拉索施加相应的位移和 速度, 然后由其数据采集系统采集拉索的反力, 得到的反 力再通过PMI带回到Matlab计算文件, 完成循环。循环过 程如图10所示。PMI将整个试验过程的数据记录并保存为 TXT文件用于试验结果分析。

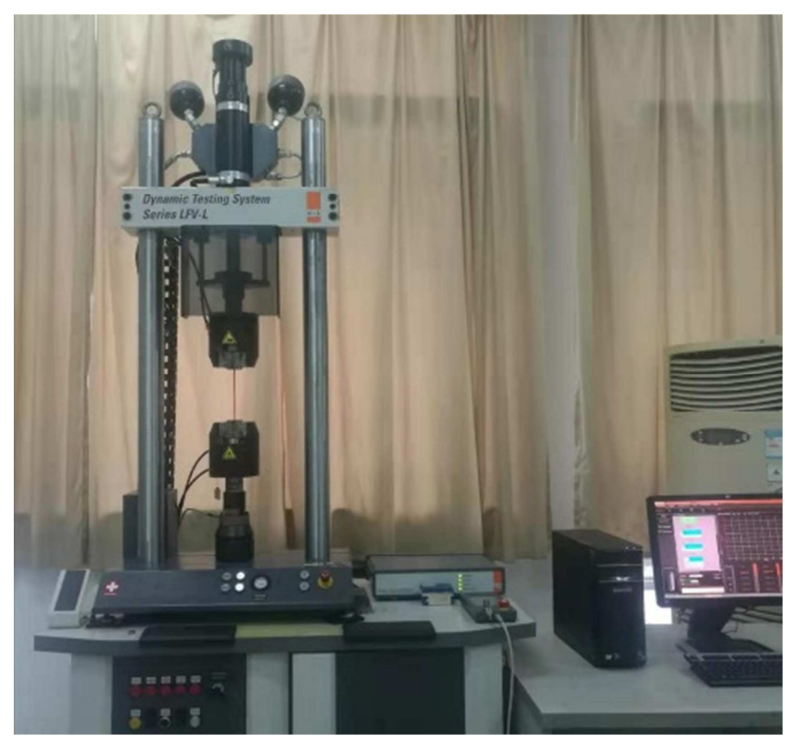

图8 LFV-10型动态多功能测试设备。

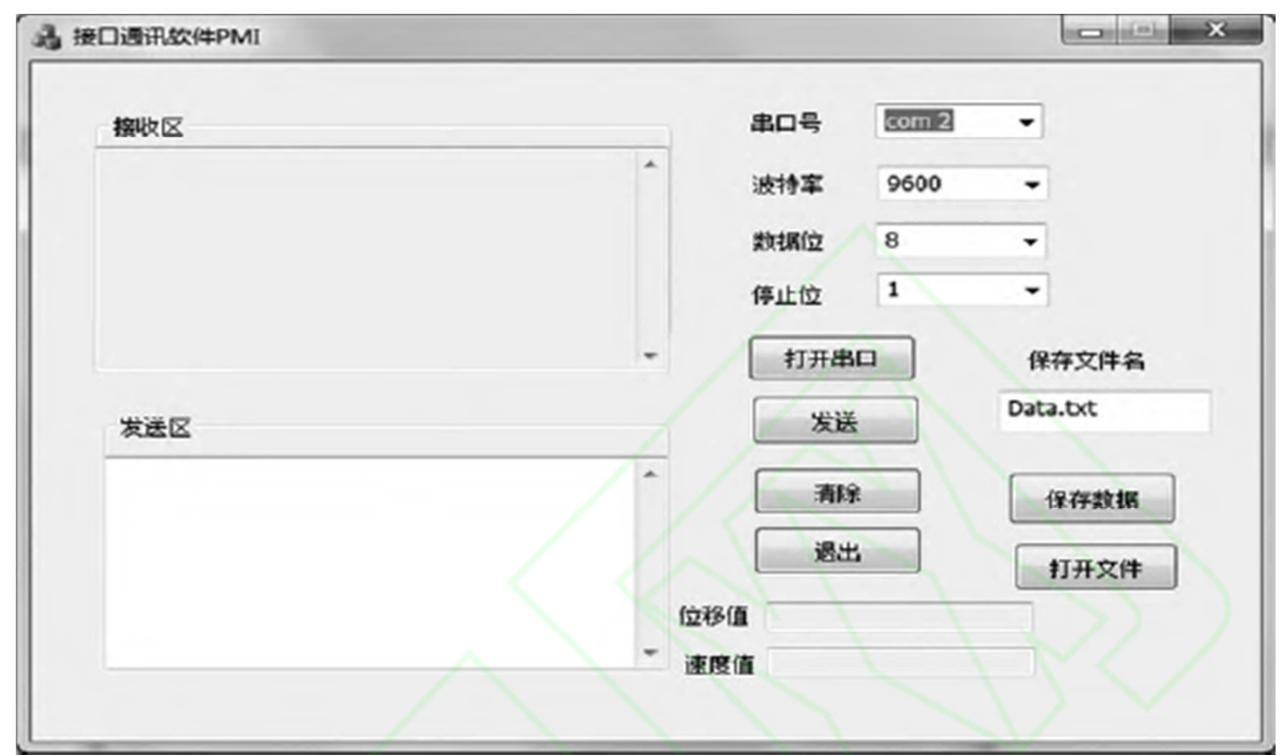

图9 PMI程序界面。 




图10 试验子结构循环过程。

\section{4. 对比试验及结果分析}

为验证混合试验的准确性, 采用数值仿真方法模拟了 钢-索结构的组合变形, 即拉索采用实验得到的力与位移 关系, 其结果与混合仿真实验结果作对比。

\section{1. 数值仿真试验}

首先在万能试验机上做 $100 \mathrm{~mm}$ 长 $4 \mathrm{~mm}$ 粗的尼龙绳索 的单轴拉伸试验 [12], 多组数据拟合得到尼龙绳索的力与 位移关系, 如图11所示。然后采用Matlab软件编写数值仿 真计算程序 $[13,14]$, 程序中运用该力与位移关系计算跨中 坚向荷载 $P$ 与杆端水平位移 $(\Delta)$ 以及拉索跨中挠度 $(f)$ 的曲线关系, 即 $P-\Delta$ 曲线和 $P-f$ 曲线。



图11 4mm粗尼龙绳索的力与位移关系。

\section{2. 混合仿真试验}

在混合仿真试验中, 试验子结构模型试验同样采用 $100 \mathrm{~mm}$ 长 $4 \mathrm{~mm}$ 粗的尼龙绳索。每个子步由数值子结构计算 得到 $x$, 由试验子结构反馈得到 $X$ 。再由 $P-X$ 关系计算 得到 $P$ 。从而以试验子结构的拉索拉力 $X$ 为桥梁, 建立 起了跨中荷载 $P$ 与杆端水平位移 $(\Delta)$ 以及拉索跨中挠度 ( $f$ ) 的关系。

\section{3. 试验结果}

图12为混合仿真试验和数值仿真试验得到的 $P-\Delta$ 曲 线, 可以看出: 两试验结果存在一定偏差, 但总体变化规 律一致; 当跨中坚向荷载小于 $600 \mathrm{~N}$ 时, 二者结果基本相等; 但在大于 $600 \mathrm{~N}$ 后, 混合仿真试验结果大于数值仿真, 不过 总体偏差小于 $5 \%$ 。原因可能是由试验设备的迟滞与卸载 行为造成, 也可能是由与足尺寸相比的缩小模型试验带来。

图13为混合仿真试验和数值仿真试验得到的 $P-f$ 曲 线, 可以看出: 两试验结果变化规律一致, 偏差较小。当 跨中坚向荷载小于 $500 \mathrm{~N}$ 时, 二者结果基本一致; 但当跨中 坚向荷载大于 $500 \mathrm{~N}$ 后, 数值仿真试验结果大于混合仿真, 不过总体偏差小于 $2 \%$ 。原因类似于 $P-\Delta$ 曲线。

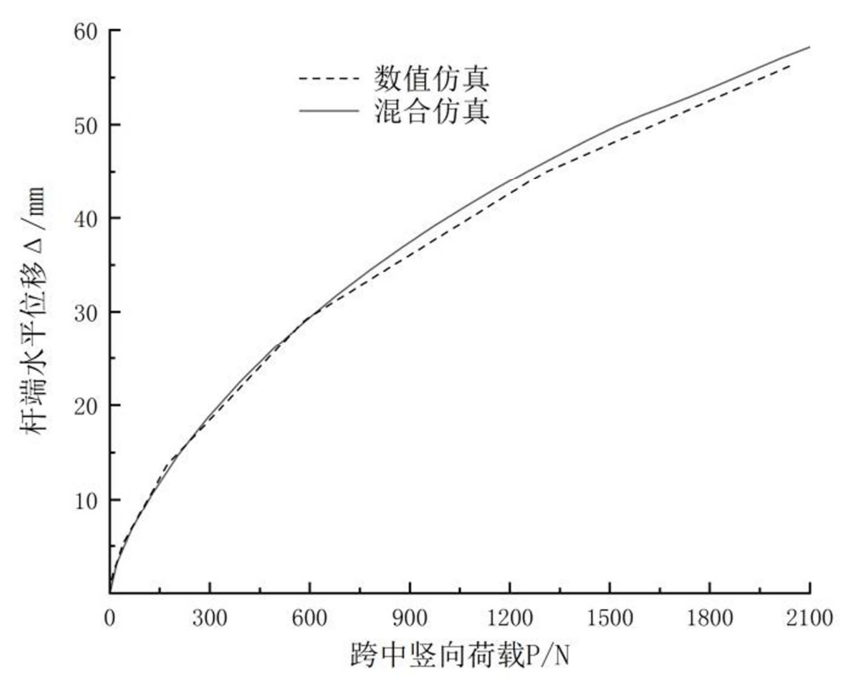

图12 $P-\Delta$ 曲线。

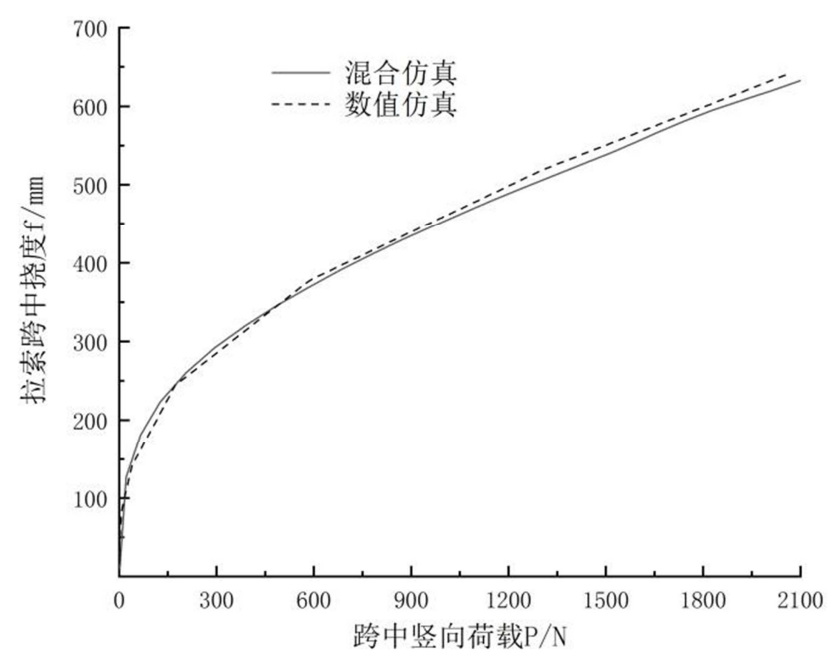

图13 $P-f$ 曲线。

\section{5. 结论}

混合仿真试验通过试验子结构与数值子结构间的数 据交换同步，实现受力状态的同步，进而模拟整体结构的 
组合变形。本文试验表明：混合仿真试验方法可以有效预 测钢-索结构的组合变形。

混合仿真试验方法可以极大地降低试验费用, 有效利 用现有设备, 对大型、复杂的结构系统, 通过简单的、主 要的、难以模拟的构件试验实现分析研究 [15]。

本文以非线性框架式钢-索结构为例, 推导了组合变 形计算方法, 并进行了结果分析。为其他形式的非线性钢 -索结构组合变形分析提供了帮助。本文中索采用的是尼 龙绳, 文中相关计算方法同样适用于钢丝绳、钢绞线等其 他类型的索。尼龙绳为弱非线性材料, 在后续研究中, 将 在强非线性材料上进一步检验混合仿真试验方法的有效 性。

\section{参考文献}

[1] 李清.悬索结构的建构及艺术呈现研究[D].大庆: 东北石油 大学土木建筑工程学院, 2013.

[2] 蓝天.中国空间结构七十年成就与展望[J].建筑结构,2019,49 (19) : 5-10.

[3] 王震, 李国强, 整体结构混合火灾试验原理及数值仿真分析 [J].防灾减灾工程学报，2012，36(03): 409-414.

[4] 董成.含速度相关型阻尼器的结构混合仿真研究[D].南京: 南京理工大学理学院, 2017.

[5] 张祁, 董成, 徐志洪.含粘弹性阻尼器结构的实时混合仿真 研究[J].南京理工大学学报, 2017, 41 (5) : 623-626.
[6] 刘振邦, 张祁, 徐志洪.含非线性拉杆的拱结构的混合仿真 研究[J].南京理工大学学报,2018, 42 (5) : 547-551.

[7] Nakashima M. Development potential and limitations of real-time online(Pseudo-dynamic) testing[J]. Philosophical Transactions of the Royal Society of London Series A-Mathematical Physical and Engineering sciences, 2001, 359(1786):1851-1867.

[8] Shao X, Enyant G. Development of a versatile hybrid testing system for seismic experimentation[J]. Experimental Techniques, 2015, 38(6):44-60.

[9] 郝伟.基于OpenFresco软件的混合试验方法[D].哈尔滨：哈 尔滨工业大学土木工程学院, 2011.

[10] 张兴虎,马否,隋壟,等.空间结构SRT混合仿真试验技术研究 [J].西安建筑科技大学学报,2014,46(03):329-332.

[11] 龙驭球, 包世华, 袁驱,等.结构力学 1 —基本教程[M].北 京: 高等教育出版社, 2012.

[12] 李阳.建筑膜材料和膜结构的力学性能研究与应用[D].上海: 同济大学土木工程学院, 2007.

[13] 徐斌.MATLAB有限元结构力学分析与工程应用[M].北京: 清华大学出版社, 2009.

[14] 张岳.MATLAB程序设计与应用基础教程（第2版）[M].北 京: 清华大学出版社, 2016.

[15] Paul L. Drazin ${ }^{a}$, Sanjay Govindjee. Hybrid simulation theory for a classical nonlinear dynamical system[J]. Journal of Sound and Vibration, 2017, 392:240-259. 